\title{
Pour un nouveau roman suédois? Quelques remarques à propos de la réception du nouveau roman en Suède
}

\author{
Cecilia Carlander \\ Université de Stockholm
}

En 1955, Alain Robbe-Grillet écrit son célèbre essai « À quoi servent les théories ? ", un texte qui non seulement discute la question de l'emploi des théories littéraires, mais qui essaie également de résumer les conditions de la littérature des années I950. Selon Robbe-Grillet, les écrivains cherchent maintenant à décrire "les nouvelles relations entre l'Homme et le monde ». Pour ce faire, il faut une nouvelle sorte de roman, un roman qui porte la marque de ces nouvelles conditions, un roman dont la forme doit rompre avec les formes déjà établies. C'est également dans ce texte que Robbe-Grillet explique que le terme de " nouveau roman " n'est rien d'autre qu' " une appellation commode englobant tous ceux qui cherchent de nouvelles formes romanesques » (Robbe-Grillet, s963:9). Bien que l'emploi de ce terme soit compliqué (et ait souvent été critiquér), le groupe d'écrivains trouve une certaine unité autour de quelques points communs : dans leur art romanesque, il s'agit surtout d'une structure narrative à laquelle manquent à la fois une intrigue traditionnelle et des possibilités pour le lecteur de s'identifier avec les personnages. Il est question d'une littérature qui ne cherche ni à informer, ni à éduquer ses lecteurs, mais qui veut explorer le monde avec ses lecteurs, ce qui exige une collaboration entre la littérature et son lectorat.

Dans l'historiographie littéraire suédoise, il n'existe jusqu'ici aucune étude qui s'intéresse pleinement au sujet de la réception des idées françaises du nouveau roman durant cette période des années I950-60. Cela est bien étonnant, vu qu'en Suède, à l'époque en question, on a d'emblée porté un intérêt considérable à ces idées françaises, et plusieurs textes importants sur le nouveau roman ont aussitôt été publiés

How to cite this book chapter:

Carlander, C. 2015. Pour un nouveau roman suédois ? Quelques remarques à propos de la réception du nouveau roman en Suède. In: Cedergren, M. et Briens, S. (eds.) Médiations interculturelles entre la France et la Suède. Trajectoires et circulations de I945 à nos jours. Pp. 205-2I6. Stockholm: Stockholm University Press. DOI: http:// dx.doi.org/ıo.I6993/bad.p. License: CC-BY 
dans la presse suédoise ${ }^{2}$. Pour cette raison, nous estimons que la question de la réception des idées du nouveau roman en Suède mérite d'être soulevée. Vu le cadre limité de cette présentation, nous devrons nous contenter de ne donner que quelques exemples choisis de la réception de ces idées en Suède - d'abord dans le débat culturel et littéraire, ensuite dans la littérature elle-même - pour discuter des possibilités d'une recherche approfondie du sujet.

\section{Les introducteurs suédois des idées françaises et le débat culturel}

\section{L'exemple Bjurström}

L'introducteur certainement le plus éminent de la littérature française en Suède à l'époque est le traducteur et écrivain Carl Gustaf Bjurström qui écrit régulièrement sur le nouveau roman, par exemple dans les essais pour le magazine littéraire de Bonniers, BLM, "Finns en fransk roman ? ( ( Y-a-t-il un roman français », I955) et « Den nya romanen » ("Le nouveau roman », I958). Il consacre également d'autres essais aux "nouveaux romanciers ", dans lesquels sont présentés des écrivains comme Michel Butor, Alain Robbe-Grillet, Claude Ollier, Nathalie Sarraute et Claude Simon. De plus, Bjurström traduit un grand nombre de ces écrivains ${ }^{3}$.

À côté de Bjurström, il faut mentionner encore quelques autres des premiers introducteurs du nouveau roman français en Suède, comme Lasse Söderberg - qui écrit un essai sur Le Voyeur d'Alain RobbeGrillet - ou encore Artur Lundkvist qui contribue avec des textes comme "Le roman français à l'heure actuelle. Les romans du soupçon » - un texte qui fait bien entendu allusion aux idées formulées par Nathalie Sarraute dans L'Ère du soupçon (1956). Un autre nom très important est celui d'Eva Alexandersson qui traduit, par ailleurs avant Bjurström, plusieurs nouveaux romans en suédois, le premier d'entre eux étant Le Voyeur (Stenögonen, 1957) de Robbe-Grillet ${ }^{4}$.

Pourtant, c'est surtout l'article de Bjurström dans le numéro d'août I95 8 de $B L M$ qui a un premier effet considérable dans le débat suédois. Les idées de Robbe-Grillet y sont résumées - tirées pour la plupart des articles écrits par Robbe-Grillet lui-même (dans L'Express, FranceObservateur, Nouvelle Revue Française, Critique ...). Bjurström présente également Robbe-Grillet comme le "chef de la nouvelle écoles " et suggère que ce dernier "repousse » le roman réaliste établi, ainsi que le roman avec une profondeur " plus ou moins psychologique ». Ainsi, 
il se fait le porte-parole de Robbe-Grillet et résume sa pensée en déclarant qu'il est temps de prendre un nouveau regard sur la réalité. Le réalisme traditionnel nous montre un monde imprégné par des mensonges, c'est-à-dire un monde conventionnel. La question se pose également de savoir s'il ne vaudrait pas mieux d'abord faire représenter la 'surface', ce qui est plus visible, pour ensuite s'intéresser aux 'profondeurs' cachées derrière ce que l'on voit facilement, et qui sont, bien évidemment, moins visibles que la 'surface'.

Dans l'essai de Bjurström, c'est donc, précisément comme en France, Robbe-Grillet qui est vu comme le chef du mouvement littéraire, et c'est certainement pourquoi les mots de ce dernier seront le plus souvent cités dans les débats à propos de l'éventualité d'un nouveau roman en Suède. Bjurström continue à attirer son attention sur la représentation littéraire de la réalité - du réel - qui est, selon Robbe-Grillet, liée à la surface : "Il faut faire représenter ce qui est, sans y mêler des forces étrangères ou impossibles à contrôler qui menacent de falsifier l'image, et décrire ce que nous voyons, sans transférer sur l'image des sentiments, des atmosphères, des théories ou des conceptions du monde. » (Bjurström, I955:640).

Après avoir discuté de la relation entre la surface et le réel, Bjurström présente les écrivains : d'abord Michel Butor et son rapport aux mythes, puis Nathalie Sarraute et Claude Simon et leur relation commune avec l'être humain. Les rubriques de l'article contribuent à donner une première interprétation des œuvres des écrivains et une analyse de leur style et de leurs thématiques. En tant qu'introduction très élaborée du nouveau roman français, l'article de Bjurström fournit un grand nombre de données importantes pour le débat suédois qui va suivre.

\section{Gustafsson et Bäckström}

Cette attention accordée au nouveau roman français contribue, comme nous avons pu le constater, à une discussion sur le roman suédois de l'époque, et on commence à se poser des questions sur l'éventualité d'un nouveau roman suédois. Par ailleurs, le roman est également le genre littéraire dont la critique littéraire suédoise discute le plus.

Un livre consacré au roman et publié au printemps I96I va contribuer à intensifier le débat, à savoir Nio brev om romanen ["Neuf lettres sur le roman »], une correspondance entre les écrivains Lars Gustafsson et Lars Bäckström ${ }^{6}$. Dans la mesure où la relation entre la littérature et le réel est la question centrale de l'ouvrage, il n'est pas 
étonnant que le nouveau roman français s'invite dans la discussion. D'emblée, dans la première lettre de Gustafsson, les idées de RobbeGrillet sont évoquées : selon lui, les idées du Français ne correspondent qu'à une nouvelle interprétation littéraire du réel ayant "la prétention erronée d'être l'image objective ", une interprétation qui ne diffère des autres que parce qu'elle est en vérité «plus pauvre, plus forcée, puisqu'elle ne représente pas la vision organique qui pousse, que l'âme elle-même exige, mais seulement - un programme littéraire. »Un roman est autre chose, continue-t-il, quelque chose qui prend forme et se déploie " entre l'âme et le monde ", chose qu'il est difficile de changer. Gustafsson est donc d'abord clairement critique vis-à-vis du programme de Robbe-Grillet. Cependant, il poursuit avec quelques lignes tout à fait semblables aux idées du nouveau roman, non seulement à celles de Robbe-Grillet mais aussi à celles de Sarraute. Cela est manifeste lorsqu'il se demande quel tort il y aurait à ce qu'une narration soit abstraite, ou encore pourquoi une histoire serait obligée d'être une histoire "vraie " avec une intrigue, des personnages et des paysages (Gustafsson \& Bäckström, I955: 7) ?

Dans sa réponse, Bäckström essaie lui-même de décrire un lac, chose qui développe sa pensée, et qui s'approche également des idées de RobbeGrillet. Selon lui, le romancier qui doit présenter le réel n'a pas à le faire d'une façon entièrement conventionnelle : ainsi, il ne devrait pas décrire un lac comme profond si la profondeur n'a pas d'importance spécifique et si elle arrive à être transmise dans la représentation. Bäckström souhaite donc que les écrivains deviennent suffisamment intelligents pour parvenir à rompre avec les conventions et s'efforcer de transmettre le réel dans leurs romans (Gustafsson \& Bäckström, I955 : I2). De même, décrire la réalité dans une société moderne devient, selon Bäckström, de plus en plus difficile, car les choses ont tellement changé que tout ce qui avait été établi - les systèmes religieux, politiques et mythiques - est maintenant à soupçonner - remarquons ici que la pensée de Bäckström ressemble à celle de Sarraute - et il souligne encore une fois qu'un art romanesque moderne ferait mieux de remettre en question les conventions. Il prend notamment pour exemple de roman non-conventionnel un roman de Gustafsson, Poeten Brumbergs sista dagar och död [ Les derniers jours et la mort du poète Brumberg ", I959]. Finalement il conclut que l'art romanesque moderne a aussi raison d'essayer, comme le fait Robbe-Grillet, de représenter une sorte d'univers commun à travers un fragment réduit mais vraiment visible de la réalité et que tout le monde puisse reconnaître. 
La correspondance entre Gustafsson et Bäckström discute également des "Lettres parisiennes " de Bjurström (les essais publiés dans BLM). De même, après avoir assisté à une conférence donnée par RobbeGrillet et Sarraute à Uppsala (à l'automne, I960), Bäckström constate que, somme toute, la littérature française a dû évoluer " à rebours " avec les idées du nouveau roman. Si le nouveau roman français s'est libéré du personnage clair et aux contours bien définis, le roman suédois devrait faire le contraire. À ce propos, Gustafsson répond qu'il vient de rencontrer l'écrivain P. O. Sundman lors de l'enregistrement d'une émission télévisée où ce dernier lui aurait dit qu'il « n'existe pas d'intrigues intérieures » chez l'homme, du moins pas en ce sens qu'il serait possible de les décrire, ce qui fait qu'on ne pourrait pas réclamer de paysages intérieurs ni d'intrigues dans un roman. Gustafsson continue, toujours à propos de cette entrevue :

[...] je réponds en disant : " Mais qui t'a fait croire qu'un roman doit contenir exactement ce que tu peux dire - ce qui est important, c'est que le roman fonctionne, et non qu'il peut être présenté comme des résultats de recherches, c'est cela, la différence entre les romans et les thèses de doctorat. " Mais Sundman allume sa pipe et fait non de la tête. De tous les théoriciens du roman dans le pays, il est le plus obstiné. (Gustafsson \& Bäckström, I955 : 86 sq.)

\section{Palm}

Avec la correspondance entre Gustafsson et Bäckström en arrière-plan, Göran Palm écrit, en I96I, un essai dans lequel il cherche à résumer l'art romanesque en Suède : "Den nya svenska romanen » [ Le nouveau roman suédois»]. Palm résume les tendances et les courants littéraires de son époque, et donne comme exemple le "nouveau regard » de Robbe-Grillet. Il compare ce regard esthétique avec celui de P. O . Sundman dans Undersökningen (L'enquête en français, publié en suédois 1958 et en français en I976), et il y trouve des ressemblances. S'il observe que le film a dû jouer un grand rôle pour Robbe-Grillet, tandis que le béhaviorisme est d'une plus grande importance pour Sundman, il veut cependant montrer que le style des deux écrivains se ressemble, car tous deux cherchent le plus souvent à décrire - sans interpréter ; l'apparition des choses de la surface est donc ce qui est recherché dans l'art de la représentation romanesque, aussi bien pour Sundman que pour Robbe-Grillet. Par ailleurs, soulignons ici qu'il s'agit, encore une fois, d'une comparaison avec Robbe-Grillet, et non pas avec d'autres écrivains considérés comme des nouveaux romanciers. 
Le regard est donc, selon Palm, commun aux deux écrivains. Palm est également d'accord avec le résumé fait par Gustafsson (dans la correspondance avec Bäckström) des tendances des représentations romanesques de l'être humain : chercher à brosser des portraits de personnages « vivants » et réalistes n'est pas toujours la meilleure méthode. Il précise ainsi que l'idée selon lui incompréhensible qui veut qu'un roman réussi contienne des êtres qui semblent vivants est insoutenable à un tel point qu'elle clôt les possibilités d'une représentation. "Les personnages romanesques sont des éléments narratifs, par principe égaux à des choses, des problèmes, des paysages. » (Palm, I96I). Pour lui, les personnages peuvent être vagues, abstraits ou morts tant qu'ils servent à quelque chose dans le roman. Le récit lui-même, dont l'intrigue se déroule dans l'âme, doit posséder une vie qui lui est bien propre.

Par ailleurs, Palm n'est pas le seul à remarquer les ressemblances entre Sundman et Robbe-Grillet. En effet, lorsque l'œuvre de Sundman est publiée en France, la critique française associe la prose de ce dernier au nouveau roman, même si l'auteur lui-même rejette ces idées, ce qui est étonnant vu le témoignage de Gustafsson (dont on a parlé plus haut $)^{7}$.

\section{Ekbom}

Un autre écrivain « clef » dans le milieu littéraire suédois de l'époque est Torsten Ekbom, qui se lance dans le débat avec l'article « Romanen som verklighetsforskning » [ «e roman comme recherche du réel »] (I962). Contrairement à ses collègues, Ekbom rappelle avant tout, plutôt que celles de Robbe-Grillet, les idées de Sarraute dans L’Ėre du soupçon pour remettre en question les formes établies du roman, surtout celles qui demandent une anecdote complète. À cet égard il est proche des idées de Sundman et de Palm quand il déclare que, dans la vie réelle, les expériences qu'il fait ressemblent rarement à des anecdotes avec des intrigues bouclées - par conséquent, pourquoi tout le temps essayer d'en avoir dans la fiction?

\section{Des traces littéraires - l'exemple de Pär Rådström}

En ce qui concerne le débat de l'art romanesque en Suède des années I950/60, nous pouvons vite constater que ce sont surtout les théories de Robbe-Grillet et de Sarraute qui intéressent les écrivains. À la suite de ce débat, on en voit aussi qui commencent à écrire dans la même veine 
du nouveau roman français. Nous en avons déjà vu deux exemples avec Lars Gustafsson et Per Olof Sundman, lequel a même été introduit en France comme un nouveau romancier ${ }^{8}$. Il reste bien évidemment encore d'autres écrivains suédois qui mériteraient une attention particulière dans ce contexte. Ici, je voudrais prendre l'exemple de l'écrivain Pär Rådström qui, autour de I960, commence à changer de style dans ses romans Sommargästerna (I960) [ "Les Invités de l'été »] et Mordet. En sörmländsk herrgårdsroman ["Le meurtre. Un roman de manoir du Södermanland»] (I962).

Les deux romans sont, en effet, dans la critique littéraire, comparés au nouveau roman français, mais ils n'ont pas, jusqu'ici été l'objet d'analyses se concentrant sur leur relation avec les écrivains français contemporains. La critique qui associe ces romans de Rådström à la littérature française de l'époque n'est pas étonnante, car il s'agit des romans avec une structure non-conventionnelle où l'intrigue est subordonnée aux impressions visuelles. Au sujet de Sommargästerna, on écrit par exemple qu'on a affaire à " une direction toute nouvelle, dont le pays natal est la France où l'on parle d'un «nouveau roman» » (Persson, 1960).

Dans aussi bien Sommargästerna que Mordet, un air soupçonneux imprègne les intrigues, ce qui ressemble parfois à des non-intrigues, voire à des anti-romans et à ce qu'avait écrit Sartre à propos du premier roman de Nathalie Sarraute (Sartre, I947). Le narrateur change souvent, d'une manière inattendue, de perspective ou de voix et le récit est parfois divisé ou même fragmentaire ${ }^{9}$. De même, la narration d'une intrigue potentielle est interrompue par des descriptions d'objets. Les dernières œuvres de Rådström (décédé jeune, en I963) s'approchent ainsi aussi bien de l'abstrait que du concret, c'est-à-dire précisément de ce qui est souvent considéré comme paradoxal dans le nouveau roman français : l'abstrait devient concret et vice versa. Pour illustrer cela, voici un extrait de Mordet. En sörmländsk herrgårdsroman :

Et soudain, je retrouve l'appétit. Je trouve également une poêle, je dois d'abord y faire bouillir de l'eau puis du lait, et je pense quand même que les œufs ont un goût étrange. Je m'assieds sur la table de la salle à manger avec mon repas sur un plateau et entends mille reproches. Tu manges sur la table de la salle à manger ? Non mais ça va pas bien, mon garçon? Tu n'es pas habillé correctement ! Maintenant Jårdsch [le prénom du protagoniste est George] va monter s'habiller correctement, qu'est-ce que le docteur dira, Anne va arranger le plateau, voilà, comme ça, et Jårdsch peut bien s'asseoir 
dans la salle à manger, d'ailleurs, il est bientôt l'heure de prendre le petit déjeuner.

Mais il fait assez froid dans la maison et je laisse durcir une moitié d'œuf sur l'assiette que j'ai rincée à l'eau froide, et il reste encore un peu d'eau. Il fait humide partout dans la maison, et je monte m'habiller mais je n'ai pas le courage de me raser et ensuite je fais un tour parmi les pièges [à souris] et trouve encore cinq souris et le plateau reste sur la table toute la matinée. Au milieu de la table de la salle à manger qui est couverte d'une nappe tissée des millions de grains de poussière. Et le jaune d'œuf pâlit dans la journée et je retrouve le pardessus que papa portait toujours en automne quand il nous surveillait, Kalle, Monsieur Johansson et moi, alors que nous sortions la barque de l'eau. Habillé de ce pardessus, je me promène dans le jardin et vois combien les choucas et les étourneaux se sont goinfrés de poires et de prunes et on entend encore le bruit des pommes qui continuent à tomber par terre. Le pommier Signe Tillisch n'est plus bon apparemment. Les pommes sont grandes et vertes mais leur peau est couverte de taches marron qui ressemblent à des croûtons. Le plateau reste sur la table toute la journée. Je n’ai plus faim (Rådström, I962: I6 sq.) ${ }^{\text {Io }}$.

Comme l'extrait ci-dessus nous le montre -extrait d'ailleurs à la fois facile à choisir, parce qu'il a été écrit dans un style représentatif du reste du roman, et difficile, parce que les choix représentatifs possibles sont tellement nombreux- les voix et les temps se mêlent chez Rådström, sans donner d'exemples concrets ni d'un intérieur profond du protagoniste, ni d'une intrigue bien précise. Ce qui reste est l'impression des objets qui, en quelque sorte, se narrent eux-mêmes, et racontent à leur tour des histoires, grâce aux associations qu'ils éveillent. Il s'agit donc bien, dans la narration, d'un style semblable à celui du nouveau roman, car ni le protagoniste, ni l'intrigue ne semblent clairs pour le lecteur, de même que les objets sont d'une valeur essentielle. Ainsi, le lecteur doit donc participer à la création du texte littéraire, ce qui est un des buts fondamentaux des écrivains du nouveau roman français ${ }^{\mathrm{II}}$. En effet, la narration de Rådström ressemble bien à ces " fragments de narration, description, souvenirs, parfois totalement discontinus, parfois juxtaposés, parfois liés au moyen d'un fondu » dont parle Jean Alter à propos du style des nouveaux romanciers français en donnant l'exemple de Claude Simon (Alter, I972: 45). Très fragmentaire, la narration peut être comparée aux images d'une caméra qui bouge de manière brusque dans la maison où se trouve le protagoniste, ce qui est aussi comparable au style des films de la nouvelle vague, le mouvement du cinéma contemporain au nouveau roman et dont les auteurs parfois étaient les mêmes, entre autres Robbe-Grillet ${ }^{\mathrm{I} 2}$. 


\section{Pour une étude de la réception du nouveau roman en Suède ? Conclusion}

Dans l'histoire littéraire française, le nouveau roman est aussitôt devenu un terme, et on en parle parfois comme d'une époque bien établie, tandis que, dans l'histoire littéraire suédoise, cela n'est pas le cas. Lorsque la critique littéraire suédoise s'est intéressée à la littérature des annés I950/60, il a surtout été question des écrivains et leurs œuvres, sans comparaisons explicites avec les idées du nouveau roman. Même si, dans ces études et ouvrages, on trouve parfois quelques aspects, idées ou commentaires qui sont liés au nouveau roman français et à son influence possible, il n'existe, jusqu'aujourd'hui, aucune étude pleinement consacrée à la réception du nouveau roman et de son esthétique en Suède.

Une cause de l'oubli de ce courant dans l'histoire littéraire en Suède pourrait se trouver dans la complexité de la définition du terme de « nouveau roman ", au sujet duquel on n'est toujours pas d'accord ou dont on n'est sûr ni du contenu, ni de l'existence. Plus un courant est difficile à cerner, voire à définir, plus son mouvement peut se traduire différemment - pourtant, on en parle aujourd'hui dans l'histoire littéraire française comme d'un courant ou d'un mouvement, et on en a beaucoup discuté en Suède dans les années I950-60. Si l'on a porté une attention aussi grande aux idées du nouveau roman en Suède à cette époque, il est remarquable que ces idées ne soient, depuis, quasiment plus discutées dans la recherche. Dans ce domaine se cache donc un véritable champ de recherche. Afin de réussir une telle étude, il faut, bien évidemment, tout d'abord cerner et définir avec plus de netteté les idées françaises, ainsi que donner un arrière-plan plus précis et plus vaste aussi bien des textes des nouveaux romanciers que de la recherche déjà faite sur ce domaine. De même, le débat culturel et littéraire suédois de l'époque doit être plus méthodiquement étudié, ainsi qu'un plus grand nombre d'exemples littéraires.

\section{Notes}

I. « Ce n'est pas un groupe, ni une école. On ne lui connaît pas de chef, de collectif, de revue de manifeste. ", (Ricardou, I973: 6). Voir également Jefferson (1980) et Ricardou \& Van Rossum-Guyon (I972).

2. Voir Lundqvist (I98 I : I 8), et Hansen (I996: 74-76).

3. Entre autres la majeure partie de l'œuvre de Claude Simon (la première traduction est celle de Tentative de restitution d'un retable baroque (Vinden, 
I96I), mais aussi des extraits des Instantanés ("Två prosastycken", BLM Io (1955), 805-807) de Robbe-Grillet, L'emploi du temps (Tidsschema, I965) ainsi que des essais du Répertoire (Sex essäer om romankonsten, I966) de M. Butor, Le Fiston (Sonen, I965) de R. Pinget, et plus tard Enfance (Innan bilden bleknat, I984) de N. Sarraute, dont Bjurström a déjà traduit des extraits des Tropismes en 1957 ( Tropismer I, II, VI, XIV », Upptakt 5 (I957): 24-25).

4. D'autres traductions d'Alexandersson : La Modification (Resa mellan kvinnor, I959) de Butor, La Jalousie (Jalusi, I960) de Robbe-Grillet, Le Planétarium (Planetarium, I96I) de Sarraute.

5. «Den nya skolans ledare » (Bjurström, I955:640).

6. La correspondance est datée entre le 29 juin I 960 et le I 6 décembre I960.

7. Voir Warme (1978) et J. Lundström (2004).

8. À propos de Sundman, voir Palm (I96I : 453 sq.), Warme (I978), Le Clec'h (I978) et Lundström (2006 : I8 I-I86).

9. Sartre écrit que Sarraute, « en laissant deviner une authenticité insaisissable [...], a mis au point une technique qui permet d'atteindre, par-delà le psychologique, la réalité humaine, dans son existence même. " (Sartre, I947 : I 5). Ce style est comparable à celui de Rådström.

Io. La traduction est la mienne.

I I. Voir Sartre (I947 : I 5), ainsi que Ricardou (I972 : I3 sq.) à propos de la détérioration du personnage. Voir également A. Jefferson ( $1980: 3)$ à propos de l'impression d'une disparition du personnage ou de l'intrigue.

I2. Voir A. Gardies (I972: I 85 sq.).

\section{Bibliographie}

Alter, J. (I972), "Perspectives et modèles ", in Ricardou J. \& Van RossumGuyon, F. (éd.). Nouveau Roman: hier, aujourd'hui (vol. I/2). Paris : Union Générale d’Editions Io/ı $8: 35-54$.

Bjurström, C. G. (I955), « Finns en fransk roman? ». BLM Io (I955) : 808-8 I 5 .

Bjurström, C. G. (I958), « Den nya romanen ». BLM 8 (I958) : 639-646.

Brundin, D. (2006), Omvägens estetik. Om Pär Rådström. Skellefteå : Norma.

Butor, M. (I960-I982), Répertoire I-V. Paris : Les Editions de Minuit.

Compagnon, A. (2002), "Nathalie Sarraute ou l'usage de l'écriture ». CRITIQUE 656/657:3-I42. 
Ekbom, T. (I962), "Romanen som verklighetsforskning ». Ord \& Bild 6 (I962) : 517-522.

Gardies, A. (I972), "Nouveau Roman et cinéma : une expérience décisive ", in Ricardou, J. \& Van Rossum-Guyon, F. (éd.). Nouveau Roman: hier, aujourd'hui (vol. I/2). Paris : Union Générale d’Editions Io/I 8 : I 8 5-I99.

Gustafsson, L. (I959), Poeten Brumbergs sista dagar och död. Stockholm : Norstedt.

Gustafsson, L. \& Bäckström, L. (I96I), Nio brev om romanen. Stockholm : Norstedt.

Hansen, P. (I996). Romanen och verklighetsproblemet. Studier i några svenska sextiotalsromaner. Stockholm / Stehag : Symposion.

Jefferson, A. (I980), The nouveau roman and the poetics of fiction. London / New York : Cambridge University Press.

Le Clec'h, G. (I978), « P. -O. Sundman, l'aigle renraciné ». Le Figaro littéraire, 27 mai.

Lundkvist, A. (I958), "Fransk roman just nu : Misstrons romaner ». MorgonTidningen, 3 I août.

Lundqvist, Å. (I98I), Från sextital till àttital. Färdvägar i svensk prosa. Stockholm : Alba.

Lundström, J. (2004), " Nouveau roman i det svenska 6o-talet. Exemplet P.O. Sundman ", in Gemzøe, A. (éd.), Fortcellingen i Norden efter I 960. Den 24. IASS-studiekonference 2002. Aalborg : I66-I7I.

- (2006), Terrängbeskrivning. P. O. Sundman, moderniteten och medmänniskan. Lund : Ellerströms.

Palm, G. (I96I), "Den nya svenska romanen ». BLM 6 (I966) : 450-459.

Persson, K. (I960), "Sommargästerna » (critique signée K.P.). Borås Tidning, 27 octobre.

Ricardou, J. (I967), Problèmes du nouveau roman. Paris : Seuil.

- (I97I), Pour une théorie du Nouveau roman. Paris : Seuil.

— (I972), "Le Nouveau Roman existe-t-il ? ", in Ricardou J. \& Van RossumGuyon F. (éd.). Nouveau Roman: hier, aujourd'bui (vol. I/2). Paris : Union Générale d’Editions ıo/ı $8: 9-20$.

- (I973), Le Nouveau Roman. Paris : Seuil.

Ricardou, J. \& Van Rossum-Guyon, F. (éd.), (I972). Nouveau Roman: hier, aujourd’hui (2 vol.). Paris : Union Générale d’Editions Io/I 8. 
Robbe-Grillet, A. (1963) [1955], "A quoi servent les théories ? ", in Pour un nouveau roman. Paris : Les Editions de Minuit.

Rådström, P. (I960), Sommargästerna. Stockholm : Norstedt.

— (I962), Mordet. En sörmländsk herrgårdsroman. Stockholm : Norstedt.

Sarraute, N. (I956), L'Ère du soupçon. Paris : Gallimard.

Sartre, J.-P. (I947), "Préface ", in Sarraute, N. (I948), Portrait d’un inconnu. Paris : Robert Marin.

Sundman, P. O. (1958), Undersökningen. Stockholm : Norstedt.

Söderberg, L. (I955), « Mätsticka och måsöga. Anteckningar kring Le Voyeur av Alain Robbe-Grillet ». Upptakt I ( I955) : 3 I-32.

Warme, L. G. (I978), "Per Olof Sundman and the French New Novel Influence or Coincidence?". Scandinavian Studies $50: 403-4$ I3. 